\title{
AMS FALL WESTERN SECTIONAL
}
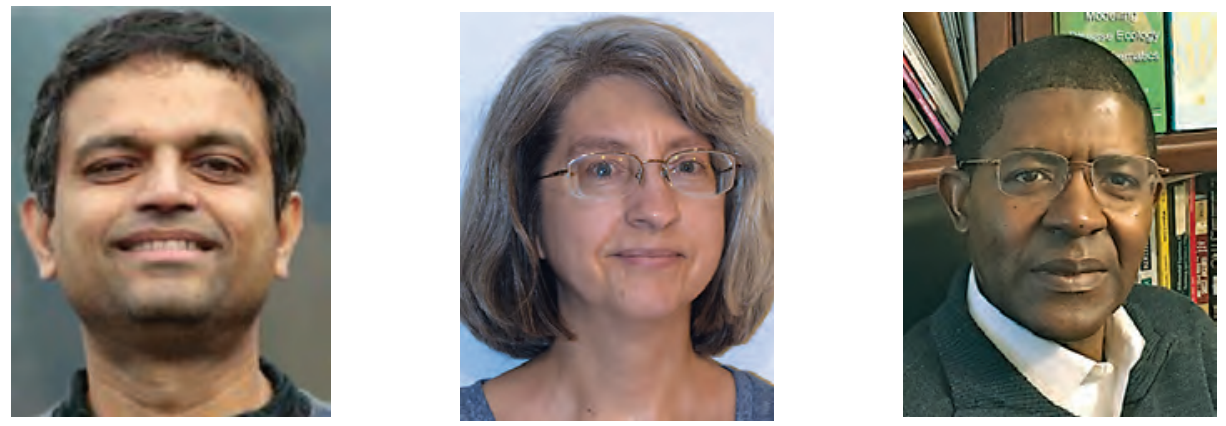

From left to right: Srikanth B. Iyengar, Sarah Witherspoon, and Abdul-Aziz Yakubu.

In this sampler, the speakers below have kindly provided introductions to their Invited Addresses for the AMS Fall Western Sectional taking place October 27-28 at San Francisco State University.

Finite Free Complexes over Polynomial Rings Srikanth B. Iyengar (University of Utah) page 1077

Derivatives, Derivations, and Hochschild Cohomology

Sarah Witherspoon (Texas A\&M University) page 1078

Population Cycles in Discrete-time Infectious Disease Models Abdul-Aziz Yakubu (Howard University) page 1079

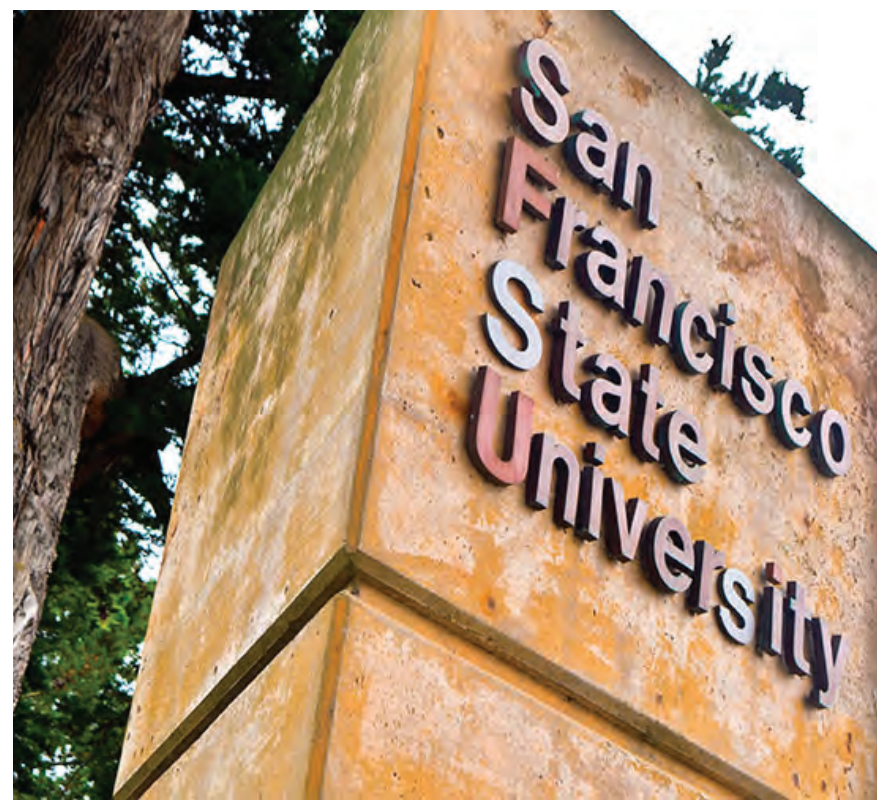

\section{EDITOR'S NOTE.}

Although we have no samplers from the AMS Central Sectional at University of Michigan, October 20-21, it also looks like a great meeting: www. ams . org/meetings/

sectiona1/2259_program.htm 1

For permission to reprint this article, please contact:

reprint-permission@ams .org.

DOI: http://dx.doi.org/10.1090/noti1792 


\section{AMS FALL WESTERN SECTIONAL}

\section{Srikanth B. Iyengar}

\section{Finite Free Complexes over Polynomial Rings}

The story I want to present begins with Hilbert. It concerns linear operators acting on a vector space $V$ over a field $k$. The action of commuting linear operators $t_{1}, \ldots, t_{d}$ on $V$ can be codified into an action of the polynomial ring $S:=k\left[x_{1}, \ldots, x_{d}\right]$ on $V$, with each $x_{i}$ acting via $t_{i}$. Said otherwise, $V$ is an $S$-module. A simple example is $V=S$, with each $x_{i}$ acting by multiplication. The free module (of rank $n$ ) is the $n$-fold direct sum, $S^{n}$, with the natural action. These are called free modules because the action is as free as possible, in that they induce no relations on the $x_{i}$. Not every module is free: consider $k$ with each $x_{i}$ acting as zero. Hilbert's Syzygy Theorem states that any $S$-module $V$ can be resolved by free $S$-modules, in the sense that there is a diagram of maps

(†) $0 \longrightarrow S^{\beta_{n}} \stackrel{\partial_{n}}{\longrightarrow} S^{\beta_{n-1}} \stackrel{\partial_{n-1}}{\longrightarrow} \cdots \stackrel{\partial_{2}}{\longrightarrow} S^{\beta_{1}} \stackrel{\partial_{1}}{\longrightarrow} S^{\beta_{0}} \longrightarrow 0$, where $\partial_{i}$ is compatible with the $S$-action, $\operatorname{Kernel}\left(\partial_{i}\right)=$ Image $\left(\partial_{i+1}\right)$ for each $i \geq 1$, and Cokernel $\left(\partial_{1}\right)=V$. The remarkable point is that only finitely many free modules are involved; indeed Hilbert proved that there exists a resolution with $n \leq d$. The module Image $\left(\partial_{i}\right)$ is called an $i$ th syzygy of $V$, so the theorem says that $V$ has a syzygy that is free. The integers $\beta_{i}$ are called the Betti numbers of $V$; these are algebraic analogues of Betti numbers in topology, and they are just as interesting as their topological counterparts. The prototypical example is a resolution of $k$ called the Koszul complex. It appears in essentially every article I have written; this one need not be an exception, so here it is for $d=3$ :

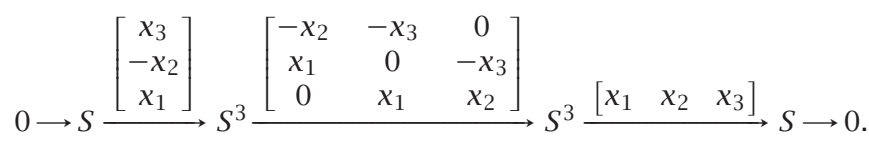

I am viewing the elements of the various free modules as column vectors. You can guess what the Koszul resolution of $k$ is for a general $d$; the Betti numbers satisfy $\beta_{i}=\left(\begin{array}{l}d \\ i\end{array}\right)$.

In the early 1970s Buchsbaum and Eisenbud conjectured that whenever the $k$-vector space $V$ is finite-dimensional, its Betti numbers satisfy $\beta_{i} \geq\left(\begin{array}{l}d \\ i\end{array}\right)$. This conjecture remains open. It implies the following.

Total Rank Conjecture. $\sum_{i=0}^{n} \beta_{i} \geq 2^{d}$.

This inequality was conjectured by Horrocks and also by Avramov. It was settled recently by Mark Walker whenever the characteristic of $k$ is not two using a beautiful argument involving Adams operations on complexes.

Srikanth B. Iyengar is professor of mathematics at the University of Utah. His email address is iyengar@math. utah. edu.

For permission to reprint this article, please contact:

reprint-permission@ams.org.

DOI: http://dx.doi.org/10.1090/noti1728
A complex is a diagram $F$ of the form $(\dagger)$ where $\operatorname{Kernel}\left(\partial_{i}\right)$ contains Image $\left(\partial_{i+1}\right)$ for each $i$; in the cases of interest to us the corresponding quotient vector space, called the homology of $F$ in degree $i$, is finite-dimensional. Resolutions of finite-dimensional modules are examples of such complexes, but many natural constructs lead to the more general kind. Experience had led us to expect that properties of resolutions of finite-dimensional modules would carry over to such $F$. In particular, it had been conjectured (by me anyway) that the Total Rank Conjecture holds also for such complexes.

Walker visited me last year, and within a few minutes of our meeting (the date, April 27, is unforgettable) we realized that between what he knew and what I had learnt recently about Lefschetz elements in exterior algebras-from a collaboration with Conca and Herbigwe could construct counterexamples to the Total Rank Conjecture for complexes. Subsequently it dawned on us that the same simple idea yields counterexamples to several other conjectures, including one due to Avramov and Buchweitz about Betti numbers of modules over complete intersection rings.

Lower bounds (almost all conjectural) similar to the Total Rank Conjecture appear also in the context of group actions on spaces. Notable among these is a conjecture of Carlsson for spaces admitting a free action of $(\mathbb{Z} / p \mathbb{Z})^{r}$, the elementary abelian $p$-group of rank $r$, where $p$ is a prime number, and one due to Halperin about spaces that admit a free action of the torus, $\left(S^{1}\right)^{r}$. Mark and I could also construct counterexamples to algebraic analogues of their conjectures. These examples challenge long-held intuition about finite free complexes and have sowed a sense of confusion in me. I'll try to convey some of this in my talk.

\section{Photo Credit}

Author photo by Tatjana Ruf, copyright MFO, used on the terms of the Creative Commons License Attribution-Share Alike 2.0 Germany.

\section{ABOUT THE AUTHOR}

Srikanth B. Iyengar is interested in commutative algebra in its various manifestations, and in particular in the modular representation theory of finite groups. He was awarded the Bessel Research Prize (2007) by the Humboldt Foundation, was a Simons Fellow in Mathematics (2012-13), and is a

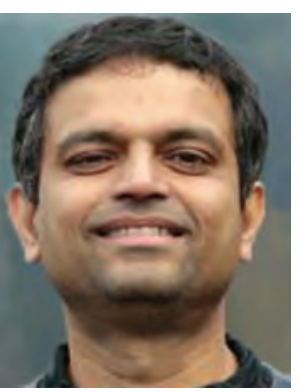

Srikanth B. Iyengar Fellow of the American Mathematical Society. 


\section{AMS FALL WESTERN SECTIONAL}

\section{Sarah Witherspoon}

\section{Derivatives, Derivations, and Hochschild Cohomology}

In the 1940s, inspired by homological methods in topology, Hochschild, Eilenberg, and Mac Lane introduced them in algebra. One outcome was Hochschild cohomology. It encodes essential information about rings, topological spaces, and categories. It has many applications, users, and developers. For example, it encodes possible deformations of an algebra, it measures coarse properties of representations via support varieties, and it determines geometric properties such as smoothness in both commutative and noncommutative settings. Yet Hochschild cohomology also has some remaining mysteries. These are the focus of much current research and of my talk.

This story begins with the Leibniz rule for differentiation of functions $f$ and $g$ on the real line:

$$
\frac{d}{d x}(f g)=\frac{d}{d x}(f) g+f \frac{d}{d x}(g) .
$$

Generalize this rule to elements of rings $R$ that are also vector spaces over fields such as $\mathbb{C}$ or $\mathbb{R}$ : A linear function $\delta$ from $R$ to $R$ is called a derivation if

$$
\delta(f g)=\delta(f) g+f \delta(g)
$$

for all $f$ and $g$ in $R$. Fundamental examples are indeed given by differentiation on rings of functions, such as polynomial rings or functions on a manifold.

The set of all derivations on $R$ forms a vector space. This vector space is not closed under composition of functions. However it is closed under a related binary operation: If $\delta$ and $\delta^{\prime}$ are two derivations on $R$, then so is $\left[\delta, \delta^{\prime}\right]$, defined as

$$
\left[\delta, \delta^{\prime}\right]:=\delta \circ \delta^{\prime}-\delta^{\prime} \circ \delta .
$$

The operation [ , ] is called a commutator or Lie bracket, and the vector space of derivations together with this bracket operation is called a Lie algebra. Vector fields on manifolds are derivations on their rings of functions, forming central examples of such Lie algebras.

More generally, consider multilinear functions from $R \times \cdots \times R$ to $R$. What is a suitable analogue of derivation? Hochschild's definition of cohomology for rings answers this question via multilinear analogues of the Leibniz rule. Hochschild cohomology spaces consist of multilinear functions obeying these rules.

Is there an analogue, for multilinear functions, of the Lie bracket on derivations? Yes! In the 1960s Gerstenhaber introduced a bilinear operation on multilinear functions, and thus on Hochschild cohomology, that is now called

Sarah Witherspoon is professor of mathematics at Texas A\&M University. Her email address is sjw@math. tamu.edu.

For permission to reprint this article, please contact:

reprint-permission@ams.org.

DOI: http://dx.doi.org/10.1090/noti1726

the Gerstenhaber bracket. Together with additional algebraic structure and fortuitous properties, Hochschild cohomology spaces are important algebraic invariants for rings.

Since its introduction by Hochschild and its further development by Gerstenhaber and others, Hochschild cohomology has found applications in many settings, for example in algebraic deformation theory, representation theory, and noncommutative geometry. For some of these applications, a deep understanding of the Gerstenhaber bracket is required. A view of Hochschild cohomology as a certain Ext group has long promised insight that is realized in recent work. In my talk I will survey some of this work as well as give an overview of Hochschild cohomology and its place in algebra.

\section{Photo Credit}

Author photo courtesy of Sarah Witherspoon.

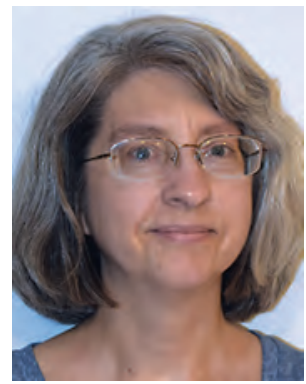

Sarah Witherspoon

\section{ABOUT THE AUTHOR}

Sarah Witherspoon's research is in noncommutative and homological algebra. She is a Fellow of the American Mathematical Society. 


\section{AMS FALL WESTERN SECTIONAL}

\section{Abdul-Aziz Yakubu}

\section{Population Cycles in Discrete-Time Infec- tious Disease Models}

ABSTRACT. The standard next generation matrix approach for calculating the basic reproduction number, an important parameter in understanding the evolution and prevention of infections, suffers from some assumptions that are not always met in real-world systems. In this talk, we explore extensions through two discrete-time infectious disease models, a SIR and an ISA $v$ model, and the different behaviors that arise.

My talk will focus on infectious disease transmission dynamics in periodic population environments. In infectious disease epidemiology, the basic reproduction number, denoted by $\mathcal{R}_{0}$, is an important threshold parameter that provides insight when designing prevention and control strategies for established infections. $\mathcal{R}_{0}$ is the average number of secondary cases produced by an infectious individual introduced into a population of susceptible individuals.

The next generation matrix (NGM) approach for defining and calculating $\mathcal{R}_{0}$ divides the population into compartments, some of them infectious. It provides an easy way to compute $\mathcal{R}_{0}$ from epidemiological reasoning and basic infectious disease model ingredients. However, for autonomous infectious disease models (without seasonality or periodicity), the NGM approach assumes the existence of a locally asymptotically stable disease-free equilibrium in the demographic equation. That is, in the absence of the disease, the population is assumed to be at a static steady state. Populations do not grow indefinitely over time, and density dependence or other factors tend to drive populations toward their carrying capacity. However, it is possible for populations to experience some fluctuations around the carrying capacity, a situation that the existing NGM approach does not address. The population fluctuations can be periodic (cyclic) or erratic (chaotic). For example, such prominent and persistent cyclic fluctuations have been observed in the abundance of some sockeye salmon populations [2].

In a recent paper, van den Driessche and Yakubu [4] used a general autonomous discrete-time epidemic model to extend the NGM approach for calculating $\mathcal{R}_{0}$ to account for period $k$ population cycles in the disease-free system, where $k \geq 1$. $\mathcal{R}_{0}<1$ implies the local asymptotic stability of the periodic disease-free population cycle and the disease goes extinct, whereas $\mathcal{R}_{0}>1$ implies the instability of the periodic disease-free cycle and the

Abdul-Aziz Yakubu is professor of mathematics at Howard University. His email address is ayakubu@howard. edu.

For permission to reprint this article, please contact: reprint-permission@ams.org.

DOI: http://dx.doi.org/10.1090/noti1727 disease persists in the population. When $\mathcal{R}_{0}<1$ and the demographic dynamics is asymptotically constant or under geometric growth (nonoscillatory, $k=1$ ), it is possible for the disease-free equilibrium point (fixed point) to be globally asymptotically stable [3]. In 2002, Elaydi and Yakubu ([1, Theorem 3]) showed that it is not possible for a disease-free population cycle with period $k \geq 2$ to be globally asymptotically stable in the "smooth" autonomous discrete-time epidemic model. In the presentation, I will apply the extended NGM approach for calculating $\mathcal{R}_{0}$ to two discrete-time infectious disease models with Ricker recruitment functions. The first model is a Susceptible-Infectious-Recovered (SIR) model with and without vaccination, and the second one is an Infectious Salmon Anemia Virus (ISAv) model [4].

The Ricker model was first used by Ricker in 1954 to study population cycles in fish. To illustrate densitydependent population cycles in a demographic equation (in the absence of a disease), in Figure 1 we consider the discrete-time demographic equation with a Ricker recruitment,

$$
S_{t+1}=f\left(S_{t}\right)+(1-d) S_{t}, \quad t \in\{0,1,2, \ldots\},
$$

with $S_{t}$ the population size of the susceptible individuals, Ricker recruitment function

$$
f\left(S_{t}\right)=r S_{t} e^{-b S_{t}},
$$

$r>0$ the intrinsic growth rate, and $b>0$ a scaling parameter. During each unit time interval, $d \in(0,1)$ is the probability of natural death for each individual, and $(1-d)$ is the probability of staying alive. Using knowledge of the population size of the susceptible individuals, $S_{t}$, at time $t$, model (1) predicts $S_{t+1}$. The model's unit of time depends on the specific application. For example, the unit of time could be a convenient time for a follow-up census.

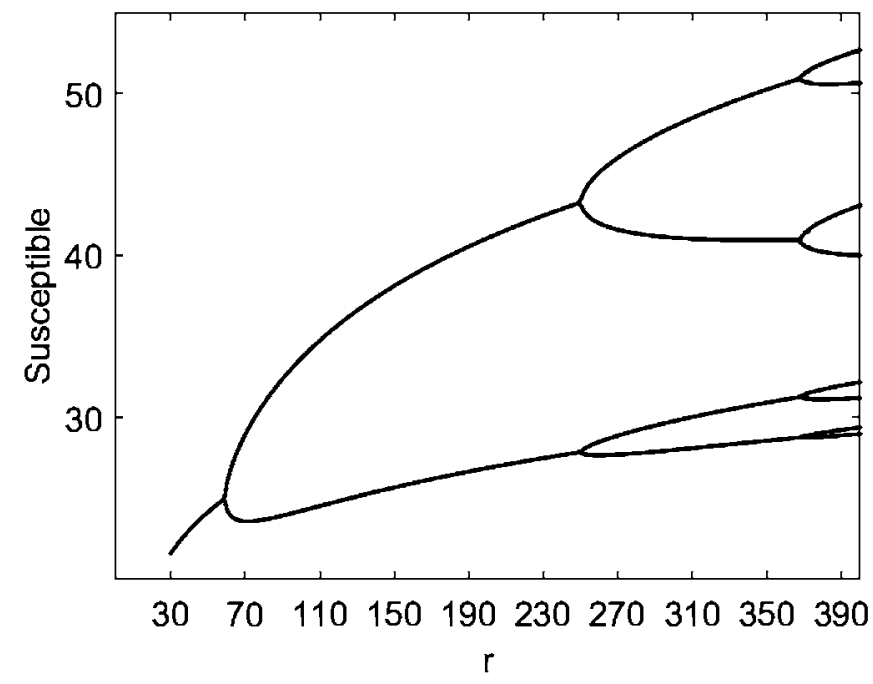

Figure 1. As the intrinsic growth rate $r$ increases, the number $S$ of susceptibles exhibits period-doubling bifurcations en route to chaos. 


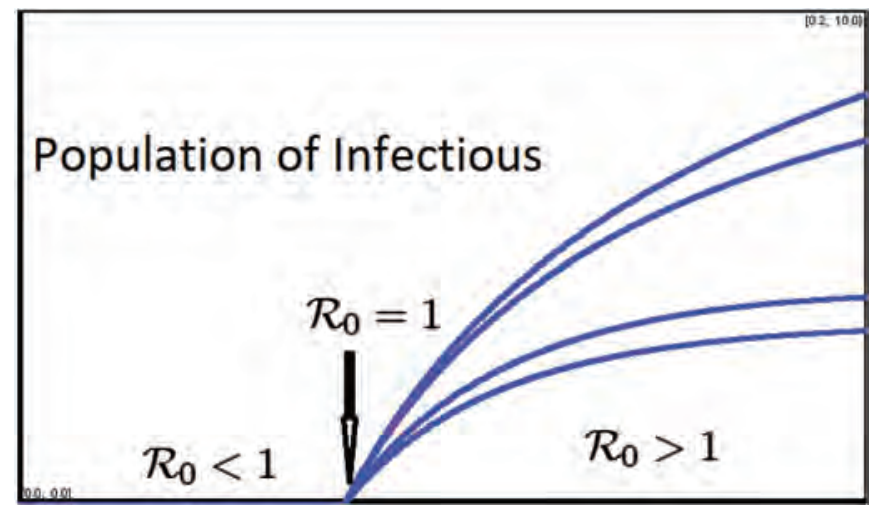

Figure 2. In this bifurcation diagram for the SIR model, as $\mathcal{R}_{0}$ increases from values less than 1 to values greater than 1 , the dynamics change from disease extinction to disease persistence on a locally asymptotically stable period 4 population cycle.

In model (1), the population goes extinct when $\mathcal{R}_{d}=$ $\frac{r}{d}<1$. However, when $\mathcal{R}_{d}>1$ the disease-free population persists on a fixed-point or cyclic or chaotic attractor. When $1<\mathcal{R}_{d}<e^{\frac{2}{d}}$, the disease-free system persists on the fixed-point attractor, $Z_{\infty}=\frac{\ln \mathcal{R}_{d}}{b}$. When $\mathcal{R}_{d}>e^{\frac{2}{a}}$, the disease-free equation (1) is known to undergo perioddoubling bifurcations en route to chaos as in Figure 1.

In general, the birth or recruitment function $f$ of model (1) is a nonlinear function that is capable of exhibiting cyclic or chaotic dynamics. An epidemic process is built on top of the demographic pattern generated by $f$. To guarantee control over the disease-free dynamics (no matter how complex they are) we assume that the disease does not affect $f$ in a significant way. This approach to model construction is quite common for continuous-time epidemic processes but less common for discrete-time epidemic models ([3], [4]).

We construct the SIR and ISA $v$ discrete-time infectious disease models on top of model (1). SIR models have been used to study a variety of diseases in humans and animals, while ISA $v$ models have been primarily used to study infectious salmon anemia, a finfish disease caused by a virus that belongs to a family of viruses called Orthomyxoviridae. ISA $v$ has caused significant mortality among salmon farms in northern Europe, Canada, Maine, and Chile. Our SIR and ISA $v$ models share a common demographic equation with a Ricker recruitment function. In the presentation, I will use the extended NGM approach to compute $\mathcal{R}_{0}$ for both models. In the SIR model, the dynamics of the Ricker demographic equation in the absence of the disease is qualitatively equivalent to the dynamics of the total population, $S+I+R$. However, in the ISA $v$ model, the dynamics of the salmon population equation, in the absence of the disease, is qualitatively different from that of the total salmon population, $S+I$. When $\mathcal{R}_{0}>1$, I will show that the period of the disease-free

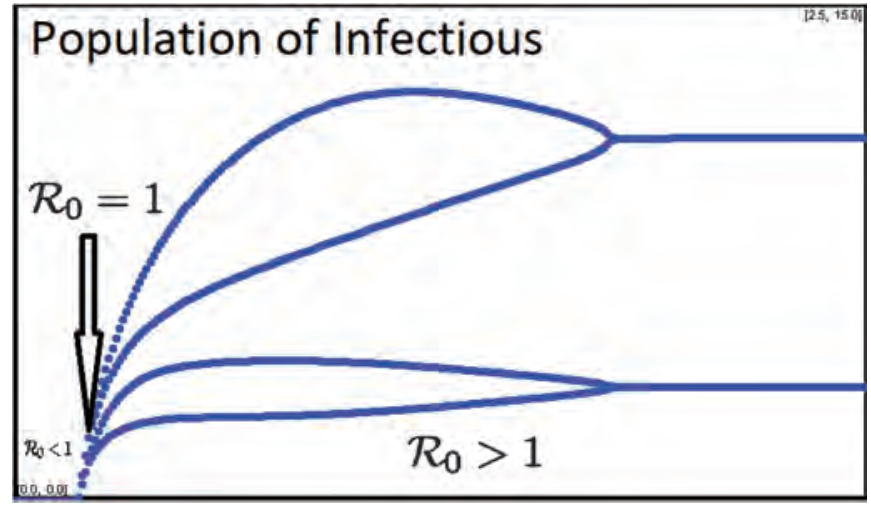

Figure 3. In this bifuration diagram for the ISAv model, as $\mathcal{R}_{0}$ increases from values less than 1 to values greater than 1 , the dynamics change from disease extinction to disease persistence on a period 4 or 2 population cycle.

susceptible population cycle in the SIR model determines the period of the infectious population cycle (see Figure 2).

Unlike the SIR model, in the ISA $v$ model, the period of the infectious salmon population can be different from that of the cyclic disease-free salmon population. In particular, I will illustrate that the ISA $v$ disease is capable of stabilizing population fluctuations in the salmon population (see Figure 3). Figure 3 shows an ISA $v$ disease-induced period-doubling reversal bifurcation, where the diseasefree salmon population is on a period 4 population cycle. The extended NGM approach accounts for periodic population cycles, and the resulting $\mathcal{R}_{0}$ computed can be used to guide prevention and control strategies in additional infectious disease environments.

\section{References}

[1] S. ELAYDI and A.-A. YAKUBU, Global stability of cycles: LotkaVolterra competition model with stocking, J. Diff. Equations Appl. 8(6) (2002), 537-549. MR1903949

[2] D. A. LeVY and C. C. Wood, Review of proposed mechanisms for sockeye salmon population cycles in the Fraser River, Bull. Math. Biol. 54 (2-3) (1992), 241-261.

[3] P. VAN DEN DRIESSChe and A -A. YAKUbU, Disease extinction versus persistence in discrete-time epidemic models, Bull. Math. Biol. (2018) https://doi .org/10 .1007/s11538-0180426-2

[4] P. VAN DEN DRIESSChe and A.-A. YAKUbU, Demographic population cycles and $\mathcal{R}_{0}$ in discrete-time epidemic models, preprint (2018) (submitted). 


\section{AMS FALL WESTERN SECTIONAL}

\section{Image Credits}

All images courtesy of Abdul-Aziz Yakubu.

\section{ABOUT THE AUTHOR}

Abdul-Aziz Yakubu's research interests include mathematical biology and dynamical systems. He coorganized several international mathematical biology workshops and conferences in Africa and was visiting professor at Botswana International University of Science and Technology, Cornell University, North East Fisheries Science Center, and The Ohio State Univer-

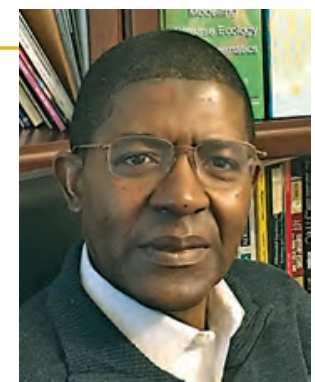

Abdul-Aziz Yakubu sity. 